\title{
Acetato de Medroxiprogesterona de Depósito como Anticoncepcional Injetável em Adolescentes
}

\author{
Depot Medroxyprogesterone Acetate as an Injectable Contraceptive for Adolescents \\ Janette Rodrigues Campos, Victor Hugo de Melo
}

\begin{abstract}
RESUMO
Objetivos: acompanhar a incidência de efeitos colaterais e aceitabilidade (taxa de continuidade) do acetato de medroxiprogesterona de depósito (DMPA) como contraceptivo injetável trimestral em adolescentes do nosso meio.

Método: quarenta adolescentes (70\% lactantes) iniciaram o uso do DMPA, tendo sido acompanhadas por uma média de 14,2 meses. As queixas espontâneas, as alterações menstruais, dados do exame fisico e exames laboratoriais foram coletados e analisados pelos testes de Wilcoxon ou McNemar.

Resultados: as queixas mais freqüentes foram cólica (16,6\%) e cefaléia $(15,2 \%)$. Os padrões menstruais predominantes foram o spotting e a oligomenorréia. Não foi observada variação significativa da pressão arterial sistólica durante o acompanhamento, mas houve uma ligeira queda nos niveis da pressão arterial diastólica, no limite da significância. Não houve alterações significativas da glicemia de jejum, e foi observada uma tendência ao aumento da taxa de hemoglobina. O ganho de peso (média de 3,9 kg em 12 meses) e a irregularidade menstrual ( $\geq 70 \%$ em qualquer retorno) foram os principais motivos para a desistência do método. Vinte e sete pacientes foram acompanhadas por 12 meses e a taxa de continuidade nessa época foi de 81,48\%.

Conclusão: o acetato de medroxiprogesterona de depósito mostrou-se um contraceptivo satisfatório para as adolescentes.
\end{abstract}

PALAVRAS-CHAVE: Anticoncepção injetável. Acetato de medroxiprogesterona. Adolescência.

\section{Introdução}

O acetato de medroxiprogesterona de depósito (DMPA) tem sido usado para contracepção por 30 milhões de mulheres em mais de 90 países desenvolvidos e em desenvolvimento ${ }^{1}$. Seu efeito contraceptivo resulta principalmente da inibição da ovulação. Além disso, o DMPA fornece eficácia contraceptiva extremamente alta por meio de: viscosidade do muco cervical aumentada, evitando assim a penetração do esperma; atrofia endometrial e alteração da motilidade da tuba uterina ${ }^{2}$.

Administrado na dose de $150 \mathrm{mg}$ a cada três meses, a eficácia contraceptiva do DMPA é de mais de $99 \%$, talvez a mais alta de todos os métodos reversiveis disponiveis. A taxa de falha de uso anual

Hospital das Clínicas da Universidade Federal de Minas Gerais - Belo Horizonte - MG

Correspondência:

Janette Rodrigues Campos

Rua Tomás Gonzaga, 802 sala 404 - Bairro de Lourdes 30180.140 - Belo Horizonte - MG associada com o DMPA é de 0,3\%, comparável à eficácia contraceptiva de três outros métodos confiáveis (esterilização tubária, implantes subdérmicos e o DIU TCu 380A) e menor que a dos contraceptivos orais (3\%). A eficácia contraceptiva do DMPA não parece ser afetada pelo peso da paciente ou pelo uso de medicações concomitantes ${ }^{3}$.

A época ótima para iniciar a contracepção com o DMPA é dentro de cinco dias do aparecimento da menstruação. Após uma injeção de 150 mg de DMPA, a ovulação não retorna por pelo menos 14 semanas $^{4}$. Embora a supressão da ovulação possa persistir raramente por até 18 meses após a última injeção, o DMPA não afeta permanentemente a fertilidade ${ }^{5}$.

Como com outros agentes progestacionais, DMPA causa supressão endometrial e atrofia. Esse efeito é manifestado clinicamente como amenorréia, sangramento irregular ou spotting ${ }^{6}$. Alterações menstruais ocorrem em quase todas as mulheres usando DMPA. Sangramento irregular imprevisivel e spotting durando 7 dias ou mais 
são comuns durante os primeiros meses de uso. Com a continuação do método, a freqüência e duração desses episódios diminuem e a amenorréia torna-se mais comum. Aproximadamente $50 \%$ das usuárias por um ano relatam amenorréia ${ }^{7}$.

O uso da medroxiprogesterona de depósito não é associado com alterações clinicamente significativas no metabolismo da glicose ou função hepática ${ }^{8,9}$. Os estudos do impacto sobre os lipídios séricos são conflitantes, alguns mostrando nenhum efeito significativo ${ }^{10}$, ao passo que outros relatam mudanças lipídicas desfavoráveis ${ }^{11}$. Resultados de um grande estudo caso-controle da OMS na Tailândia, México e Quênia reasseguram que o uso da medroxiprogesterona de depósito não aumenta o risco de carcinomas da mama, endometrial, ovariano e cervical ${ }^{12-15}$. O estudo conclui que o medicamento reduz dramaticamente o risco de câncer endometrial, por pelo menos 8 anos após sua suspensão.

Uma variedade de efeitos colaterais menores, embora incomuns, pode ocorrer em usuárias de DMPA, como: cefaléia, tonteira, intumescimento do abdome ou mamas, alterações do humor (incluindo depressão e libido reduzida) e alopécia. Durante os primeiros 2 anos de uso de DMPA, um ganho de peso de aproximadamente 2 a $4 \mathrm{~kg}$ é mais comum que a perda de peso ${ }^{16}$.

Como o uso a longo prazo de DMPA reduz a perda sanguínea menstrual, os niveis de hemoglobina freqüentemente aumentam levemente em mulheres usando esse método ${ }^{17}$.

Pesquisas mais recentes buscam esclarecer o efeito do DMPA sobre a densidade óssea. A preocupação é que o hipoestrogenismo relativo causado pela droga aumente a reabsorção óssea e o conseqüente risco de fraturas. Enquanto há um estudo mostrando que o uso prolongado de DMPA pode afetar adversamente a densidade óssea em mulheres jovens (entre 18 e 21 anos) ${ }^{18}$, um casocontrole com ex-usuárias de DMPA na pós-menopausa concluiu que quaisquer efeitos residuais da droga na densidade óssea nesse periodo são pequenos e portanto improváveis de terem qualquer impacto no risco de fratura ${ }^{19}$.

Adolescentes são freqüentemente excelentes candidatas para a contracepção com DMPA ${ }^{20}$. Elas geralmente têm problemas para aderir aos anticoncepcionais orais ou métodos de barreira e gostam da privacidade associada com o injetável. Muitas adolescentes, quando procuram cuidado médico, já estão grávidas. Como elas podem ter os mesmos fatores contribuindo para uma nova gravidez, é necessário que a anticoncepção no pósparto seja pronta, eficaz, segura - inclusive não interferindo na lactação -, conveniente e reversível. O acetato de medroxiprogesterona de depósito atende a todos esses requisitos ${ }^{16}$.

O objetivo deste experimento foi acompanhar a incidência de efeitos colaterais (queixas, alterações menstruais, alterações do peso e pressão arterial) e aceitabilidade (taxa de continuidade) do acetato de medroxiprogesterona como contraceptivo em adolescentes do nosso meio.

\section{Pacientes e Métodos}

Foram submetidas a anamnese, exame clinico, exame ginecológico e exames complementares as pacientes adolescentes freqüentadoras da Clínica de Planejamento Familiar do Hospital das Clínicas - UFMG e referidas de outros hospitais e centros de saúde, que preenchiam os critérios necessários para inclusão neste experimento nãocontrolado. Este estudo teve a aprovação prévia pela Comissão de Ética em Pesquisa do Hospital das Clinicas da UFMG.

Foram admitidas no estudo 40 adolescentes, com idade variando de 13 a 19 anos. A idade média foi 17 anos. Apenas 10\% das pacientes eram nuligestas. Setenta e cinco por cento das pacientes haviam tido um parto e $15 \%$ haviam tido 2 partos. Só uma paciente relatou um aborto prévio. Ao início do uso do DMPA, 70\% das pacientes estavam amamentando. A renda familiar de $75 \%$ das adolescentes era menor que 5 salários mínimos ao início do estudo.

Inicialmente, a paciente foi submetida à coleta de dados segundo protocolo, a um exame clínico e ginecológico completo (com citologia oncótica do colo uterino), hemograma, glicemia de jejum, dosagem de $\beta$-HCG, coagulograma e ultra-sonografia pélvica. Após essa avaliação, se considerada apta para inclusão no trabalho, a paciente assinou o termo de consentimento pós-informação e recebeu a primeira dose de $150 \mathrm{mg}$ do DMPA em uma só injeção intramuscular, no músculo deltóide ou glúteo. Recebeu também um calendário menstrual, juntamente com instruções para preenchimento. Foi orientada a retornar após um mês e subseqüentemente a cada três meses, na época da nova injeção de DMPA, ou a qualquer momento que necessitasse.

A cada retorno, a paciente foi ouvida quanto às queixas espontâneas e submetida a um questionário de acompanhamento, visando o levantamento de efeitos colaterais e intercorrências, além de seu desejo de se manter usuária do método. Seu calendário menstrual ou suas anotações sobre o ciclo menstrual foram observadas e transcritas para o seu prontuário. O padrão menstrual foi classificado segundo os seguintes critérios: amenorréia - ausência de qualquer sangramento menstrual por pelo menos 90 dias seguidos; oligomenorréia - menstruações a intervalos >35 dias e <90 dias; polimenorréia - menstruações a intervalos < 25 dias; spotting - sangramento leve, com uso de no máximo um absorvente/dia; 
hipermenorréia - sangramento menstrual prolongado, durando mais de oito dias; menorragia sangramento menstrual aumentado (mais de quatro absorventes/dia). As pacientes foram submetidas à medida de pressão arterial, peso corporal e, se necessário, a exame físico e exames complementares.

Após a terceira dose do medicamento, cada paciente das que usaram pelo menos três doses, foi submetida aos mesmos exames laboratoriais do início do estudo. O uso do DMPA foi interrompido por desejo da paciente em qualquer dose.

A coleta de dados foi feita entre agosto/ 1995 e setembro/1997. Foram admitidas novas usuárias para o método até abril/1997. O tempo médio de acompanhamento foi de 14,2 meses de uso do medicamento. No total, foram seguidos 567 meses de uso de DMPA e foram aplicadas 191 injeções de DMPA nesse período.

O teste não-paramétrico de Wilcoxon para amostras pareadas foi utilizado neste trabalho para as comparações de peso corporal, pressão arterial, hemoglobina e glicemia de jejum, considerandose os resultados nas avaliações realizadas com cada paciente. Este é o teste mais apropriado, já que o estudo caracteriza-se por comparações pareadas e há um pequeno universo de dados que não são distribuídos normalmente. $O$ teste de McNemar para amostras pareadas foi utilizado para avaliar a significância dos aumentos proporcionais de amenorréia no primeiro retorno, comparado ao $3^{\circ}, 4^{\circ}$ e 5o retorno, já que nesta avaliação se consideram as mesmas pacientes em momentos diferentes. Todas as comparações cujo valor de $\mathrm{p}$ (probabilidade de significância) foi menor que $5 \%(0,05)$ foram consideradas diferenças estatisticamente significativas.

A taxa de continuidade foi calculada considerando-se o número de pacientes que se mantiveram usuárias do método dentre aquelas seguidas a cada retorno. O número de pacientes acompanhadas variou porque foram admitidas novas usuárias de agosto de 1995 até abril de 1997. O cálculo foi feito a partir de um tempo de seguimento de seis meses. Ressaltamos que, como as pacientes entraram no estudo em tempos diferentes, o número de meses de seguimento não é uniforme. Embora a média de seguimento com uso do medicamento seja de 14,2 meses, houve pacientes que entraram no estudo 5 meses antes do encerramento.

\section{Resultados}

As queixas relacionadas ao DMPA não foram muito freqüentes durante o acompanhamento. Destacam-se as queixas de cólica e cefaléia, ambas citadas por cerca de $15 \%$ das pacientes, considerando-se a mediana dos sete momentos de avaliação. As queixas de diminuição da libido e depressão foram pouco freqüentes (média $<3 \%$ ), mas aparecem na Tabela 1 por sua freqüência em estudos da literatura. O termo "outras" refere-se a uma variedade de queixas com incidência média $<3 \%$, como dor torácica, tremores, dor epigástrica, dispareunia, constipação intestinal, diarréia, dismenorréia, etc.

Tabela 1 - Distribuição das queixas espontâneas entre as usuárias de DMPA.

\begin{tabular}{lcc}
\hline Queixa & Média \% & Mediana \% \\
\hline Cólica & 16,6 & 15,2 \\
Cefaléia & 15,2 & 15,2 \\
Mastalgia & 7,4 & 9,1 \\
Nervosismo & 6,9 & 6,7 \\
Corrimento & 6,3 & 5,1 \\
Tonteira & 5,0 & 5,6 \\
Distensão abdominal & 4,3 & 5,6 \\
Náuseas & 5,8 & 3,0 \\
Aumento de peso & 4,4 & 3,0 \\
Prurido vaginal & 3,8 & 2,8 \\
Queda de cabelo & 3,5 & - \\
Diminuição da libido & 1,4 & - \\
Depressão & 0,4 & - \\
Outras & 13,9 & 12,1 \\
\hline
\end{tabular}

O aparecimento de alguma irregularidade menstrual foi freqüente, ocorrendo em mais de $70 \%$ das pacientes em qualquer retorno. Especificando-se essa irregularidade do padrão menstrual, nota-se que o spotting foi o mais presente, sendo que, com exceção do primeiro retorno, foi relatado por mais de $50 \%$ das pacientes em todo o período de acompanhamento. A oligomenorréia foi também muito freqüente $(52,9 \%)$ e a amenorréia foi o único padrão que mostrou uma tendência de crescimento ao longo do tempo (Figura 1). A menorragia foi o padrão menos relatado $(3,7 \%)$, e nenhuma paciente necessitou de internação ou curetagem uterina por sangramento aumentado. A polimenorréia $(27,6 \%)$ e a hipermenorréia $(31,5 \%)$ foram padrões de distribuição intermediária, sem mostrarem qualquer tendência.

Avaliando-se a tendência de crescimento do padrão amenorréia, observou-se que o aumento do primeiro para o quinto retorno foi estatisticamente significativo ( $\mathrm{p}=0,034$, teste de McNemar). Isso significa que houve uma tendência para o aumento da ocorrência de amenorréia após um ano de uso do DMPA. Analisaram-se também as diferenças do primeiro retorno em relação ao terceiro e quarto retornos, mas nesses casos a diferença não foi significativa $\left(1^{\circ} \rightarrow 3^{\circ}: \mathrm{p}=0,48,1^{\circ} \rightarrow\right.$ $\left.4^{\circ}: p=0,26\right)$. O sexto retorno não foi avaliado devido ao pequeno tamanho da amostra. 


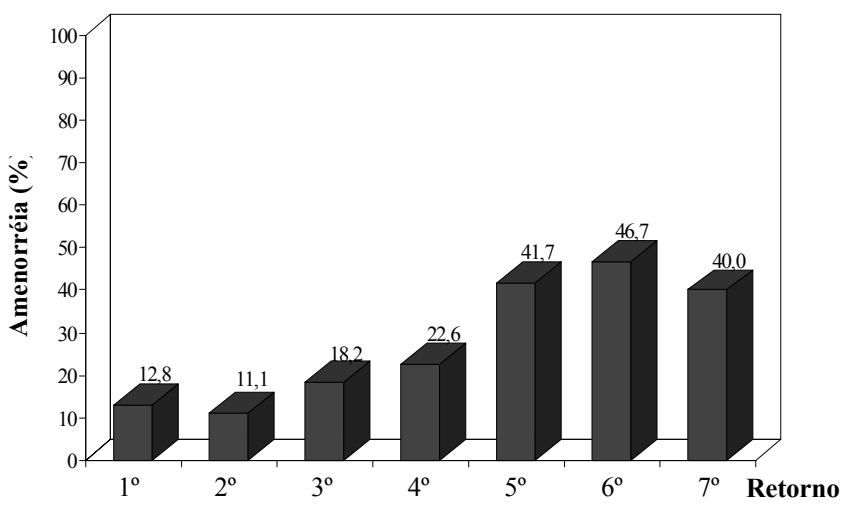

Figura 1 - Porcentagem de pacientes em amenorréia (ausência de sangramento por 90 dias ou mais) em cada retorno.

Em relação ao peso corporal inicial, observou-se uma variação entre 38,5 e 91,0 quilos, com média igual a 55,9 quilos. O peso médio mantevese em geral estável, mas aumentos estatisticamente significativos, porém suaves, foram observados no quarto e quinto retornos (de $55,9 \mathrm{~kg}$ para 57,5 e $59,8 \mathrm{~kg}$, respectivamente) e uma diminuição no sexto retorno de aproximadamente $1,7 \mathrm{~kg}$, segundo o teste de Wilcoxon. No sétimo retorno observou-se o menor peso e a menor variabilidade, mas não foi, contudo, uma diferença significativa, provavelmente pelo pequeno número de pacientes presentes nessa fase do estudo.

$\mathrm{Na}$ tentativa de se explicar a diminuição significativa de peso observada no sexto retorno, podese avaliar o comportamento do peso retirando-se da análise três pacientes que desistiram do uso do DMPA após o quinto retorno devido ao aumento excessivo de peso. Suspeita-se que houve contribuição desses casos nas diferenças observadas. A análise excluindo-se essas pacientes mostra que as médias e medianas do quarto e quinto retornos aproximaram-se mais dos valores iniciais e o valor de $\mathrm{p}$ do teste de Wilcoxon foi $>0,05$, apesar de não atingir $0,10(0,08$ na comparação com o quarto retorno e 0,053 no quinto retorno).

A pressão arterial sistólica (PAS) também mostrou um comportamento estável durante o período estudado e não se observou qualquer diferença significativa ao longo do tempo em relação ao nível pressórico no início do estudo, cuja média foi $99,9 \mathrm{mmHg}$.

Em relação à pressão arterial diastólica (PAD), não ocorreram grandes variações nas médias e a mediana foi a mesma em todos os retornos. Não houve, a rigor, diferença significativa, mas os valores de p para 5ㅇ, 6으 e 7으 retornos foram próximos de 0,05 , sugerindo uma tendência à diminuição da PAD ao longo do tempo de uso do DMPA.

Foi observado um aumento significativo na taxa de hemoglobina ( $\mathrm{p}<0,001$, teste de Wilcoxon). A média inicial foi $13,3 \mathrm{~g} \%$ e na reavaliação subiu para $14,1 \mathrm{~g} \%$. Os desvios-padrão foram muito bai- xos $(0,7$ para a hemoglobina inicial e 0,8 para a hemoglobina na reavaliação), o que indica que a taxa de hemoglobina é uma variável de comportamento estável. Nesses casos, diferenças sutis são suficientes para observar-se uma significância no teste de comparação.

A taxa de glicemia de jejum apresentou uma ligeira elevação média na reavaliação realizada após a terceira dose (de 81,7 para $85,3 \mathrm{mg} / \mathrm{dL}$ ), mas não houve significância estatística $(p=0,088$, teste de Wilcoxon). Pode mostrar, no entanto, uma tendência ao aumento da taxa, apesar de mantida nos niveis normais.

Quarenta adolescentes iniciaram o uso do DMPA neste estudo e houve apenas uma perda de seguimento. Treze pacientes desistiram do método até o fechamento da análise: quatro pacientes após uma dose, uma paciente após duas doses, duas pacientes após três doses, duas pacientes após quatro doses e quatro pacientes após cinco doses. A taxa de continuidade em cada dose é apresentada na Tabela 2. Vinte e sete adolescentes foram acompanhadas por 12 meses (cinco doses) e a taxa de continuidade nessa época foi de $81,48 \%$.

Tabela 2 - Taxa de continuidade a cada dose de DMPA.

\begin{tabular}{ccccc}
\hline $\begin{array}{c}\text { Tempo de } \\
\text { seguimento meses }\end{array}$ & $\begin{array}{c}\text { No. } \\
\text { doses }\end{array}$ & $\begin{array}{c}\text { No. } \\
\text { pacientes }\end{array}$ & \multicolumn{2}{c}{$\begin{array}{c}\text { Taxa de } \\
\text { continuidade }\end{array}$} \\
\hline 6 & 3 & 39 & $33 / 39$ & $84,62 \%$ \\
9 & 4 & 35 & $29 / 35$ & $82,86 \%$ \\
12 & 5 & 27 & $22 / 27$ & $81,48 \%$ \\
15 & 6 & 18 & $13 / 18$ & $72,22 \%$ \\
18 & 7 & 14 & $10 / 14$ & $71,43 \%$ \\
\hline
\end{tabular}

As principais causas para desistência do método foram o aumento de peso ( 6 pacientes) e a irregularidade menstrual (4 casos), na forma de hipermenorréia, polimenorréia e spotting. Outras causas foram: diminuição da libido (3 pacientes); objeção do parceiro (2 pacientes); motivo religioso, queda de cabelos, vasectomia do parceiro, desejo de engravidar e de menstruar (1 paciente cada). Algumas pacientes citaram mais de um motivo.

A avaliação da eficácia não foi um dos objetivos deste estudo, pela já conhecida alta eficácia da droga. Não houve qualquer caso de gravidez durante o acompanhamento.

\section{Discussão}

As queixas espontâneas mais frequentes foram cólica e cefaléia, com uma incidência média de $16,6 \%$ e $15,2 \%$, respectivamente. Schwallie 
e Assenzo ${ }^{17}$, estudando 3.857 usuárias de DMPA, relataram como mais freqüentes a cefaléia $(17,1 \%)$, o desconforto abdominal $(13,4 \%)$ e o nervosismo $(10,8 \%)$. Outras queixas tiveram freqüência menor que $10 \%$, como aconteceu neste estudo. Nenhuma paciente, no entanto, desistiu do método por essas queixas mais freqüentes.

A irregularidade menstrual foi muito freqüente entre as usuárias adolescentes de DMPA. Houve maior prevalência de spotting e oligomenorréia sobre os outros padrões neste estudo e a tendência para a amenorréia foi crescente, o que condiz com a literatura ${ }^{7}$. A incidência de $41,7 \%$ de amenorréia aos 12 meses é um pouco menor do que a relatada por Belsey ${ }^{7}$, que foi de aproximadamente $50 \%$.

As médias e medianas do peso aumentaram significativamente no quarto e quinto retornos e, em seguida, declinaram significativamente no sexto retorno. Essa queda era esperada por ter sido o ganho de peso uma das causas da desistência do método em seis pacientes (no total, foi o motivo mais importante para a interrupção do método). A desistência de três pacientes após cinco doses de DMPA (quinto retorno) por aumento de peso contribuiu para a diminuição significativa do peso médio no sexto retorno. A média de ganho de peso em 12 meses (cinco doses - quinto retorno) foi de $3,9 \mathrm{~kg}$. Não obstante, três pacientes perderam peso (perda de 2,4 kg, 6,3 kg e 7,9 $\mathrm{kg})$, dentre as acompanhadas por 12 meses.

O aumento médio de peso relatado pela OMS em 1.216 usuárias de sete países foi de $1,5 \mathrm{~kg}$ em um ano ${ }^{21}$. Enquanto a maioria dos estudos mostra que as usuárias de DMPA pesam mais que as mulheres do grupo-controle, os estudos mais detalhados e cuidadosamente controlados não observaram ganho de peso durante o primeiro ano de uso de DMPA. Esses estudos certamente indicam que o ganho de peso não é inevitável durante o uso de DMPA, embora alguns dos outros dados sugiram que o uso de DMPA ou MPA possa estar associado a apetite aumentado. As recomendações-padrão a respeito da ingestão nutricional e energética apropriada e o exercício regular são benéficos para o controle do peso, além de trazer beneficios para a saúde a longo prazo, e essas recomendações deveriam ser dadas a todas as mulheres em uso de DMPA ${ }^{22}$.

O comportamento da PAS, que não variou significativamente com o DMPA, está de acordo com os achados de um grande estudo multicêntrico da $\mathrm{OMS}^{21}$. A tendência observada de diminuição da PAD ao longo do tempo com o DMPA, no limite da significância, condiz com os achados de Paiva ${ }^{23}$, que observou decréscimo tanto da PAS como da PAD.

Só 31 pacientes tinham os dois valores de glicemia para serem comparados, pois cinco abandonaram o método antes da terceira dose e quatro não tiveram um ou os dois resultados para comparação. Apesar da ligeira elevação da glicemia de jejum média, o teste de Wilcoxon não mostrou significância estatística. Isso pode ter ocorrido por ter sido a reavaliação um pouco precoce (foi feita após a terceira dose). A literatura tem vários estudos testando a tolerância à glicose e/ou os níveis de insulina em usuárias de $\mathrm{DMPA}^{8,9}$. A maioria mostra evidência de tolerância alterada à glicose e niveis de insulina aumentados nas usuárias de DMPA. Neste estudo, a glicemia de jejum foi inicialmente pedida para a exclusão da paciente diabética e depois optou-se por uma comparação após a terceira dose do medicamento. Não foi estudada a tolerância à glicose.

A ligeira elevação da hemoglobina, estatisticamente significativa já nessa reavaliação após a terceira dose, está de acordo com a literatura, que mostra melhora dos niveis hematimétricos em usuárias de DMPA ${ }^{17}$. Foram retiradas da análise algumas pacientes com leve anemia à entrada no estudo ( $\mathrm{Hb}<12 \mathrm{~g} / \mathrm{dL})$, que fizeram uso de sulfato ferroso durante o acompanhamento. Além disso, algumas pacientes não foram avaliadas por terem desistido do método antes da terceira dose.

A adesão ao método foi muito satisfatória. A taxa de continuidade em nove meses foi de $82,86 \%$, próxima à descrita por Harel et al. ${ }^{24}$ em 78 adolescentes, que foi de $78 \%$. Nesse estudo, o maior motivo dado para a interrupção do método foi o sangramento irregular, que foi a segunda causa para a desistência entre as adolescentes do nosso meio. O ganho de peso, maior razão citada para a desistência pelas nossas adolescentes, foi a segunda maior causa citada no estudo de Harel et al. ${ }^{24}$.

A taxa de continuidade em um ano foi de $81,48 \%$, assemelhando-se à taxa de 50 a $80 \%$ para adultas $^{25}$.

Concluiu-se que o acetato de medroxiprogesterona de depósito mostrou-se um método contraceptivo satisfatório para as adolescentes, em sua maioria puérperas, por sua alta eficácia, grande adesão ao método, possibilidade de uso em lactantes, efeitos colaterais toleráveis e alterações menstruais sem gravidade.

\section{SUMMARY}

Purpose: to evaluate the incidence of side effects and acceptance (continuity rate) of depot medroxyprogesterone acetate (DMPA) as an injectable three-monthly contraceptive given to adolescents in our milien.

Method: forty adolescents (70\% lactating) started to use DMPA and were followed-up for a mean of 14.2 months. Spontaneous complaints, menstrual changes, physical examination and laboratory data were collected and studied using Wilcoxon or McNemar tests.

Results: the most frequent complaints were abdominal pain (16.6\%) and headache (15.2\%). Predominant menstrual patterns were spotting and oligomenorrhea. Significant variation of the systolic blood pressure was not observed 
during the follow-up. There was a slight fall in the levels of diastolic blood pressure, at the limit of significance. Significant deviations from baseline regarding fasting glucose were not noted, but the mean hemoglobin concentration tended to increase. Weight gain (mean $3.9 \mathrm{~kg}$ at 12 months) and menstrual irregularity (occurred in more than $70 \%$ of all visits) were the main reasons for discontinuation of the method. Twenty-seven patients were accompanied during 12 months and the continuity rate at that time was $81.5 \%$.

Conclusion: depot medroxyprogesterone acetate is a satisfactory contraceptive method for adolescents.

KEY WORDS: Injectable contraception. Medroxyprogesterone acetate. Adolescence.

\section{Referências}

1. Lali P, Chandra L, Gupta RP. Serum immunoglobulin levels during contraceptive use of depotmedroxyprogesterone acetate in Indian women: a preliminary study. Contraception 1996; 53:363-5.

2. Kaunitz AM. Long-acting injectable contraception with depot medroxyprogesterone acetate. Am J Obstet Gynecol 1994; 170:1543-9.

3. Kaunitz AM. Long-acting contraceptive options. Int J Fertil Menopausal Stud 1996; 41:69-76.

4. Fotherby K, Koetsawang S, Mathrubutham M. Pharmacokinetic study of different doses of DepoProvera. Contraception 1980; 22:527-36.

5. Schwallie PC, Assenzo JR. The effect of depomedroxyprogesterone acetate on pituitary and ovarian function, and the return of fertility following its discontinuation: a review. Contraception 1974; 10:181-202.

6. Cullins VE. Noncontraceptive benefits and therapeutic uses of depot medroxyprogesterone acetate. J Reprod Med 1996; 41 Suppl:428-33.

7. Belsey EM. Vaginal bleeding patterns among women using one natural and eight hormonal methods of contraception. Contraception 1988; 38:181-206.

8. Kamau RK, Maina FW, Kigondu C, Mati JK. The effect of low-oestrogen combined pill, progestogenonly pill and medroxyprogesterone acetate on oral glucose tolerance test. East Afr Med J 1990; 67:550-5.

9. Virutamasen $\mathrm{P}$, Wongsrichanalai C, Tangkeo P, Nitichai Y, Rienprayoon D. Metabolic effects of depot medroxyprogesterone acetate in long-term users: a cross sectional study. Int J Gynaecol Obstet 1986; 24:291-6.

10.Garza-Flores J, De la Cruz DL, Valles de Bourges V, et al. Long-term effects of depotmedroxyprogesterone acetate on lipoprotein metabolism. Contraception 1991; 44:61-71.

11. Chen FP, Lee N, Soong YK. Changes in the lipoprotein profile in postmenopausal women receiving hormonal terapy. Effects of natural and synthetic progesterone. J Reprod Med 1998; 43:568-74.

12.Breast cancer and depot-medroxyprogesterone acetate: a multinational study. WHO collaborative study of neoplasia and steroid contraceptives. Lancet 1991; 338:833-8.

13.Depot-medroxyprogesterone acetate (DMPA) and risk of endometrial cancer. WHO collaborative study of neoplasia and steroid contraceptives. Int J Cancer 1991; 49:186-90.

14.Depot-medroxyprogesterone acetate (DMPA) and risk of epithelial ovarian cancer. WHO collaborative study of neoplasia and steroid contraceptives. Int J Cancer 1991; 49:191-5.

15.Depot-medroxyprogesterone acetate (DMPA) and risk of invasive squamous cell cervical cancer. WHO collaborative study of neoplasia and steroid contraceptives. Contraception 1992; 45:299-312.

16.Kaunitz AM, Rosenfield A. Injectable contraception with depot medroxyprogesterone acetate. Current status. Drugs 1993; 45:857-65.

17. Schwallie PC, Assenzo JR. Contraceptive useefficacy study utilizing medroxyprogesterone acetate administered as an intramuscular injection once every 90 days. Fertil Steril 1973; 24:331-9.

18.Scholes D, Lacroix AZ, Ott SM, Ichikawa LE, Barlow WE. Bone mineral density in women using depot medroxyprogesterone acetate for contraception. Obstet Gynecol 1999; 93:233-8.

19.Orr-Walker BJ, Evans MC, Ames RW, Clearwater JM, Cundy T, Reid IR. The effect of past use of the injectable contraceptive depot medroxyprogesterone acetate on bone mineral density in normal post-menopausal women. Clin Endocrinol (Oxf) 1998; 49:615-8.

20.Kaunitz AM, Mishell DR Jr. Progestin-only contraceptives: current perspectives and future directions. Dialogues Contracept 1994; 4:1-10.

21.Said S, Omar K, Koetsawang S, et al. A multicentered phase III comparative clinical trial of depot medroxyprogesterone acetate given threemonthly at doses of $100 \mathrm{mg}$ or $150 \mathrm{mg}$ : 1 . Contraceptive efficacy and side effects. Contraception 1986; 34:223-35.

22. Westhoff C. Depot medroxyprogesterone acetate contraception. Metabolic parameters and mood changes. J Reprod Med 1996; 41Suppl:401-6.

23.Paiva LHSC. Avaliação da performance do acetato de medroxyprogesterona injetável como anticoncepcional e efeitos sobre o peso corporal, pressão arterial, ciclo menstrual e histologia endometrial [tese]. Campinas: Universidade de Campinas; 1993.

24.Harel Z, Biro FM, Kollar LM. Depo-Provera in adolescents: effects of early second injection or prior oral contraception. J Adolesc Health 1995; 16:379-84.

25.Fraser IS, Weisberg E. A comprehensive review of injectable contraception with special emphasis on depot medroxyprogesterone acetate. Med J Aust 1981; 1 Suppl:3-19. 